\title{
Enhancing Insulated Conjugated Polymer Fluorescence Quenching by Incorporating Dithia[3.3]paracyclophanes
}

\author{
Ryan Lillis, ${ }^{\dagger}$ Maximillian R. Thomas, ${ }^{\dagger}$ Manikandan Mohanan, Nagarjuna Gavvalapalli*
}
Department of Chemistry, and Institute for Soft Matter Synthesis and Metrology, Georgetown University, Washington D.C. 20057

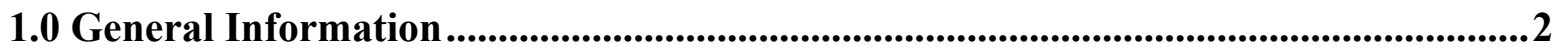

2.0 General Reaction Scheme for the Synthesis of Paracyclophane Monomer: ...............3

3.0 General Procedure for Glaser-Hay Polymerization ............................................................4

4.0 Gel Permeation Chromatography (GPC) Traces of Polymers ......................................6

5.0 Non-Normalized UV-Vis Spectra ....................................................................................

6.0 Molar Extinction Coefficient Determination Plots ........................................................ 7

7.0 Thin-Film Absorption Spectrum ...........................................................................12

8.0 Non-Normalized Emission Spectrum ...............................................................................12

9.0 Quantum Yield Measurements..................................................................................13

10.0 Stern-Volmer Plots of Copolymers......................................................................................18

11.0 Atomic Coordinates of ACP-PCP-ACP Trimer.....................................................20

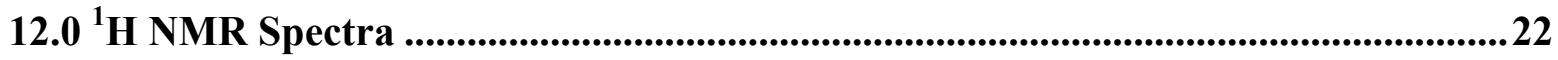




\subsection{General Information}

Unless otherwise stated, reactions were performed in oven-dried glassware fitted with rubber septa under nitrogen atmosphere and were stirred with Teflon-coated magnetic stirring bars. Reagents used for polymer synthesis were purchased from Fisher, Acros, Alfa Aesar and Sigma Aldrich. All air or moisture-sensitive reactions were performed under nitrogen atmosphere using standard Schlenk techniques. Thin layer chromatography was performed using Silicagel $60 \mathrm{~F}-254$ precoated plates $(0.25 \mathrm{~mm})$ and visualized by UV irradiation, $\mathrm{KMnO}_{4}$ stain and other stains. Silica gel of particle size 230-400 mesh was used for flash chromatography. Unless otherwise stated, all starting materials and reagents were used without further purification.

${ }^{1} \mathrm{H}$ and ${ }^{13} \mathrm{C}$ NMR spectra were recorded on Varian 400-MR NMR. Chemical shifts are reported in $\delta(\mathrm{ppm})$ relative to the residual solvent peak $\mathrm{CDCl}_{3}: 7.26$ for ${ }^{1} \mathrm{H}$; and $\mathrm{CDCl}_{3}$ : 77.36 for ${ }^{13} \mathrm{C}$; Coupling constants $(J)$ are expressed in Hertz $(\mathrm{Hz})$. Splitting patterns are designated as $\mathrm{s}$ (singlet), br(broad signal), $\mathrm{d}$ (doublet), $\mathrm{t}($ triplet), dd(doublet of doublets), $\mathrm{dt}$ (doublet of triplets), dq(doublet of quartets), m(multiplet), and q(quartet). UV-vis absorption spectra were recorded on Agilent Technologies Cary Series 5000 UV-vis-NIR Spectrophotometer. Fluorescence absorption spectra were recorded on Horiba Scientific Fluoromax-4 Spectrophotometer. Molecular weight measurements of polymers were performed by gel permeation chromatography (GPC) on Agilent Technologies 1260 Infinity. The column chromatography of UV active compounds was performed on Biotage Isolera one 3.0 unless otherwise specified.

The fluorescence quenching studies of polymers were performed in the solution state by adding different concentrations of quencher (TCNQ) in $\mathrm{v} / \mathrm{v}$ to the $13.3 \mu \mathrm{M}$ solution of polymer solution in chloroform. 


\subsection{General Reaction Scheme for the Synthesis of Paracyclophane Monomer:}

The compounds $( \pm)-\mathbf{1 2}$ and $( \pm)-\mathbf{1 3}$ were prepared according to a modified literature procedure

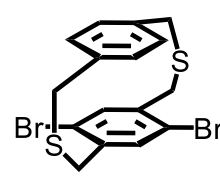

11

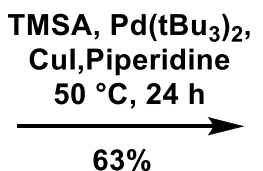

$63 \%$

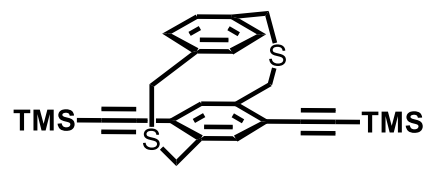

12
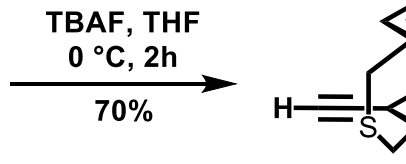

13

Synthesis of bis(trimethylsilylethynyl)[3.3]dithia-paracyclophane (12)

In an oven dried Schlenk flask dibromo paracyclophane 11 (100 mg, $0.23 \mathrm{mmol}, 1$ equiv.) was taken and brought into the glove box, bis(tri-tert-butylphosphine)palladium(0) (6 mg, $0.01 \mathrm{mmol}, 5 \mathrm{~mol} \%$ ) and copper (I) iodide ( $2 \mathrm{mg}, 0.01 \mathrm{mmol}, 5 \mathrm{~mol} \%$ ) were added along with dry and degassed piperidine $(4 \mathrm{~mL})$. Then TMS-acetylene $(200 \mu \mathrm{L}, 1.4 \mathrm{mmol})$ was added and the reaction mixture was stirred at $80^{\circ} \mathrm{C}$ for 24 hours. The crude reaction mixture was evaporated, extracted with dichloromethane $(2 \times 20 \mathrm{~mL})$, dried over $\mathrm{Na}_{2} \mathrm{SO}_{4}$, filtered through cotton, and reevaporated. A chromatographic column was prepared by creating a slurry of silica gel with 1:50 triethylamine/ $n$-hexane. The column was flushed with $n$-hexane and the crude product was separated chromatographically with $n$-hexane as an eluent. Recrystallization in $n$-hexane yielded 12 as a white solid (69 mg, 63\% yield). ${ }^{1}$ H NMR (400 $\left.\mathrm{MHz}, \mathrm{CDCl}_{3}\right) \delta: 7.05(\mathrm{~s}, 2 \mathrm{H}), 7.02(\mathrm{~s}, 4 \mathrm{H}), 4.29(\mathrm{~d}, J=16 \mathrm{~Hz}, 2 \mathrm{H}), 3.82(\mathrm{q}, 4 \mathrm{H}), 3.54(\mathrm{~d}, J=$ $16 \mathrm{~Hz}, 2 \mathrm{H}), 0.33(\mathrm{~s}, 18 \mathrm{H})$

\section{Synthesis of bis(ethynyl)[3.3]dithia-paracyclophane (13)}

TMS-acetylated paracyclophane 12 (69 mg, $0.15 \mathrm{mmol}, 1$ equiv.) was taken in THF (3 mL) and cooled to $0^{\circ} \mathrm{C}$. Tetrabutylammonium fluoride $(1.0 \mathrm{M}$ in THF, $450 \mu \mathrm{L}, 0.45 \mathrm{mmol})$ was added and the reaction mixture was stirred for 2 hours at the same temperature. The reaction was quenched by addition of $\mathrm{H}_{2} \mathrm{O}$. The organic layer was separated through a separatory funnel and dried over $\mathrm{Na}_{2} \mathrm{SO}_{4}$. The crude product was purified by column chromatography on silica gel with $\mathrm{CH}_{2} \mathrm{Cl}_{2} / n$-hexane gradient $(20 \%-60 \% \mathrm{DCM})$ as an eluent to yield 3 as a white solid (34 mg, 70\% yield). ${ }^{1} \mathbf{H}$ NMR (400 $\mathrm{MHz}^{\left.\mathrm{CDCl}_{3}\right)} \delta: 7.14(\mathrm{~s}, 2 \mathrm{H}), 7.06(\mathrm{~d}, J=8$ $\mathrm{Hz}, 2 \mathrm{H}), 7.02$ (d, $J=8 \mathrm{~Hz}, 2 \mathrm{H}), 4.30(\mathrm{~d}, J=16 \mathrm{~Hz}, 2 \mathrm{H}), 3.87(\mathrm{~d}, J=16 \mathrm{~Hz}, 2 \mathrm{H}), 3.79(\mathrm{~d}, J$ $=16 \mathrm{~Hz}, 2 \mathrm{H}), 3.59(\mathrm{~d}, J=16 \mathrm{~Hz}, 2 \mathrm{H}), 3.43(\mathrm{~s}, 2 \mathrm{H})$ 


\subsection{General Procedure for Glaser-Hay Polymerization}

Compund numbers should match main text. The copolymers CPs 1-4 were prepared according to a modified literature procedure?

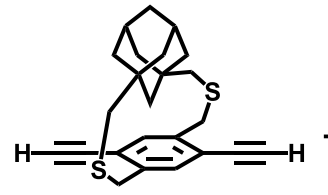

(士)-8

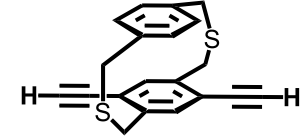

(士) -13

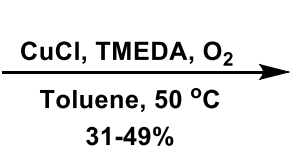

$31-49 \%$

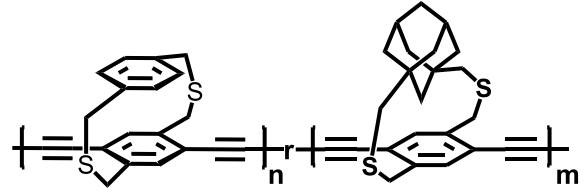

CP 1-4

\section{Synthesis of CPs 1-4}

Diacetylene paracyclophane $( \pm)-13(15 \mathrm{mg}, 0.046 \mathrm{mmol}, 0.1$ equiv.) and diacetylene adamantanocyclophane $( \pm)-8$ (160 mg, $0.42 \mathrm{mmol}, 0.9$ equiv.) were taken in toluene (18 $\mathrm{mL}$ ), and copper (I) chloride ( $24 \mathrm{mg}, 0.25 \mathrm{mmol}, 0.5$ equiv.) was added. The flask was bubbled with air for $2 \mathrm{~min}$. N,N,N',N'-tetramethylethylenediamine (40 $\mathrm{L}, 0.25 \mathrm{mmol}$ ) was then added and the reaction mixture was stirred at $50{ }^{\circ} \mathrm{C}$ until precipitate started forming. The suspension was precipitated in methanol $(100 \mathrm{~mL})$ and filtered. The suspension was centrifuged at $4500 \mathrm{rpm}$ for 15 minutes and the supernatant was decanted. To the precipitate was then added $50 \mathrm{~mL}$ diethyl ether and the suspension was centrifuged at $4500 \mathrm{rpm}$ for 15 minutes. The supernatant was decanted and the precipitate was collected. The crude polymer was purified by Soxhlet with methanol for 48 hours followed by chloroform for another 96 hours to obtain the soluble fraction. CPs 2-4 were synthesized following similar protocol, modifying the loading percent of $( \pm)-\mathbf{1 3}$ as needed.

CP-1: ( $76.3 \mathrm{mg}, 49 \%$ yield), ${ }^{1} \mathbf{H}$ NMR (400 MHz, $\mathrm{CDCl}_{3}$ ) $8: 7.68$ (brs), 7.36 (brs), 7.187.04 (m), 4.33-4.00 (m), 3.99-3.61 (m), 3.61-3.31 (d,), 2.73 (brs), 2.22-2.03 (brs), 2.02-1.73 (m), 1.68-1.38 (m), 1.34-1.08 (m), 1.05-0.87 (m), -0.03 (brs), -0.15 (brs).

CP-2: (64.5 mg, 37\% yield), ${ }^{1} \mathbf{H}$ NMR (400 MHz, $\left.\mathrm{CDCl}_{3}\right)$ $)$ : 7.68 (brs), 7.36 (brs), 7.18-7.04 (m), 4.33-4.00 (m), 3.99-3.61 (m), 3.61-3.31 (d), 2.73 (brs), 2.22-2.03 (brs), 2.02-1.73 (m), $1.68-1.38$ (m), 1.34-1.08 (m), 1.05-0.87 (m), -0.03 (brs), -0.15 (brs). 
CP-3: (56.8 mg, 33\% yield), ${ }^{1} \mathbf{H}$ NMR (400 MHz, $\left.\mathrm{CDCl}_{3}\right)$ $\delta: 7.68$ (brs), 7.36 (brs), 7.18-7.04 (m), 4.33-4.00 (m), 3.99-3.61 (m), 3.61-3.31 (d), 2.73 (brs), 2.22-2.03 (brs), 2.02-1.73 (m), $1.68-1.38$ (m), 1.34-1.08 (m), 1.05-0.87 (m), -0.03 (brs), -0.15 (brs).

CP-4: $\left(55 \mathrm{mg}, 31 \%\right.$ yield), ${ }^{1} \mathbf{H}$ NMR (400 MHz, $\mathrm{CDCl}_{3}$ ) $\delta: 7.68$ (brs), 7.36 (brs), 7.18-7.04 (m), 4.33-4.00 (m), 3.99-3.61 (m), 3.61-3.31 (d), 2.73 (brs), 2.22-2.03 (brs), 2.02-1.73 (m), $1.68-1.38$ (m), 1.34-1.08 (m), 1.05-0.87 (m), -0.03 (brs), -0.15 (brs). 


\subsection{Gel Permeation Chromatography (GPC) Traces of Polymers}

Figure S1
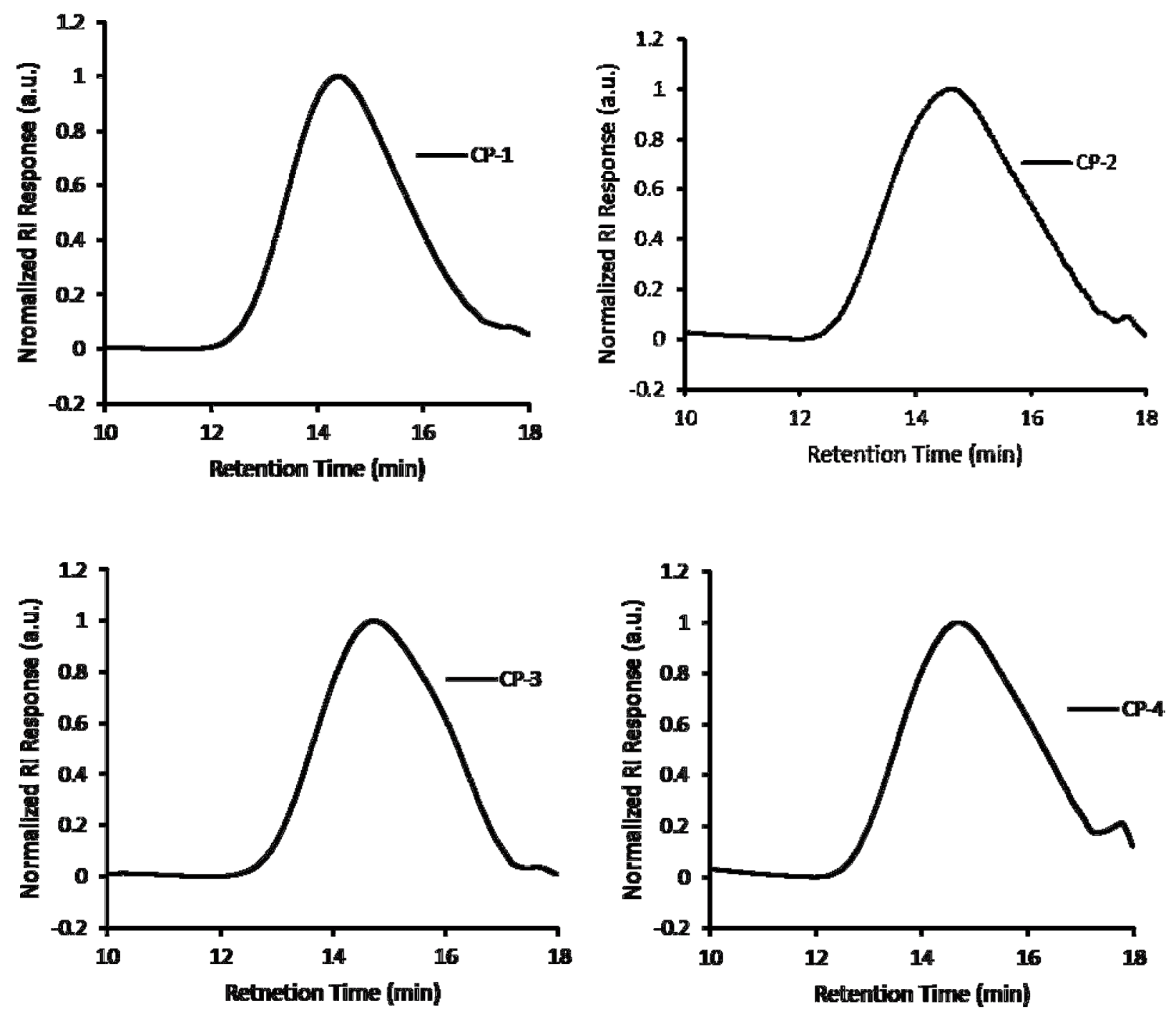


\subsection{Non-Normalized UV-Vis Spectra}

Figure S2: UV-Vis of copolymers at $32 \mu \mathrm{M}$ in $\mathrm{CHCl}_{3}$

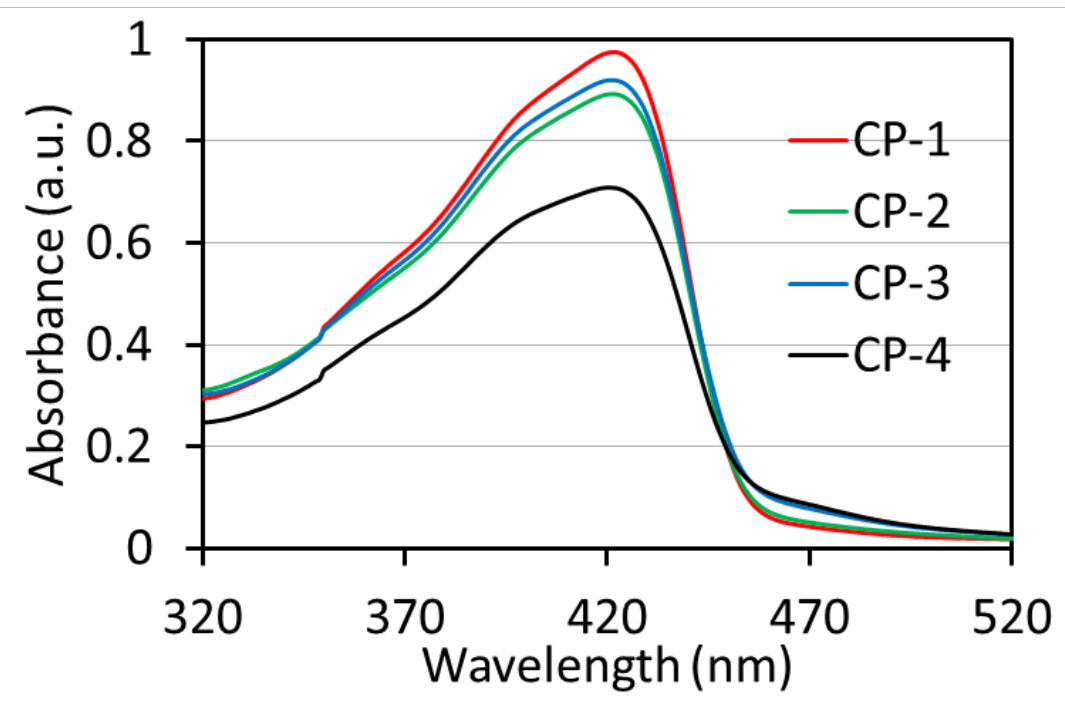

\subsection{Molar Extinction Coefficient Determination Plots}

Figure S3: CP-1

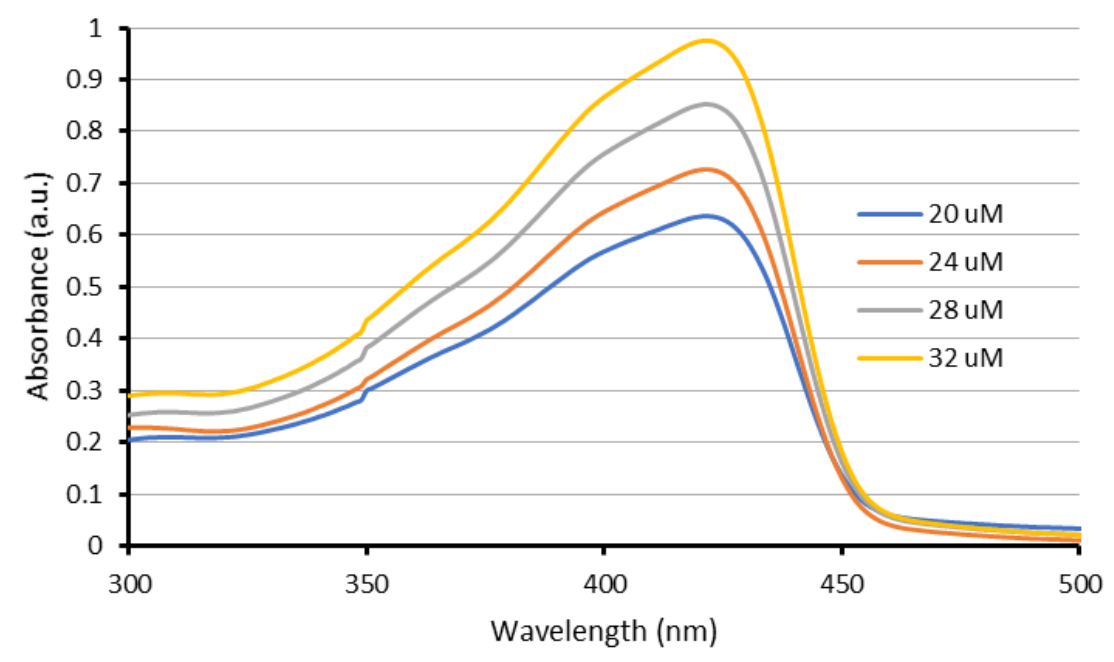


At $\lambda_{\text {max }}$

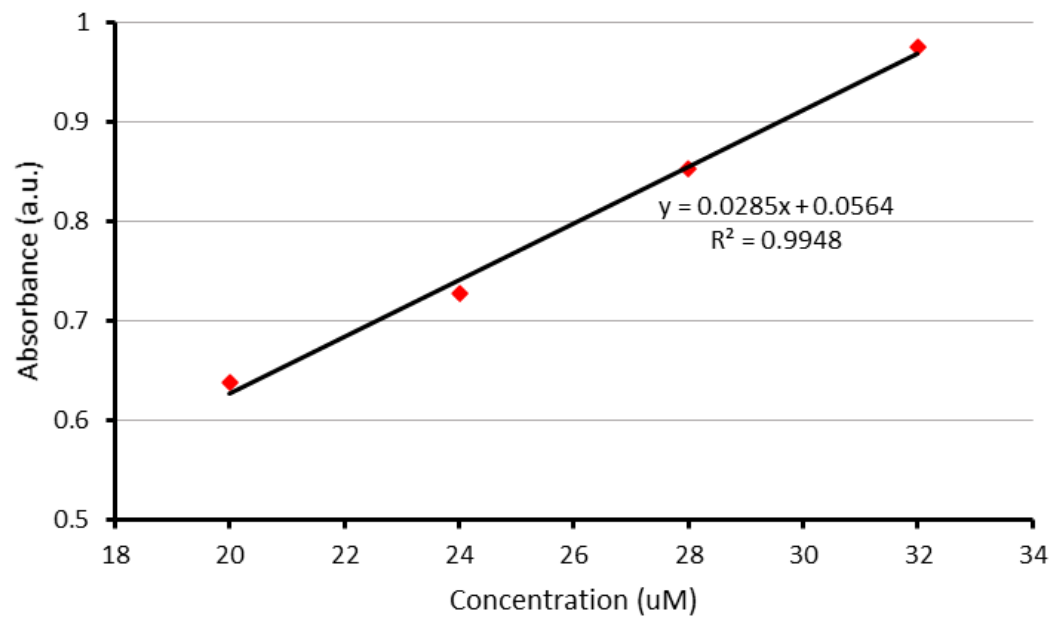

At 472 nm

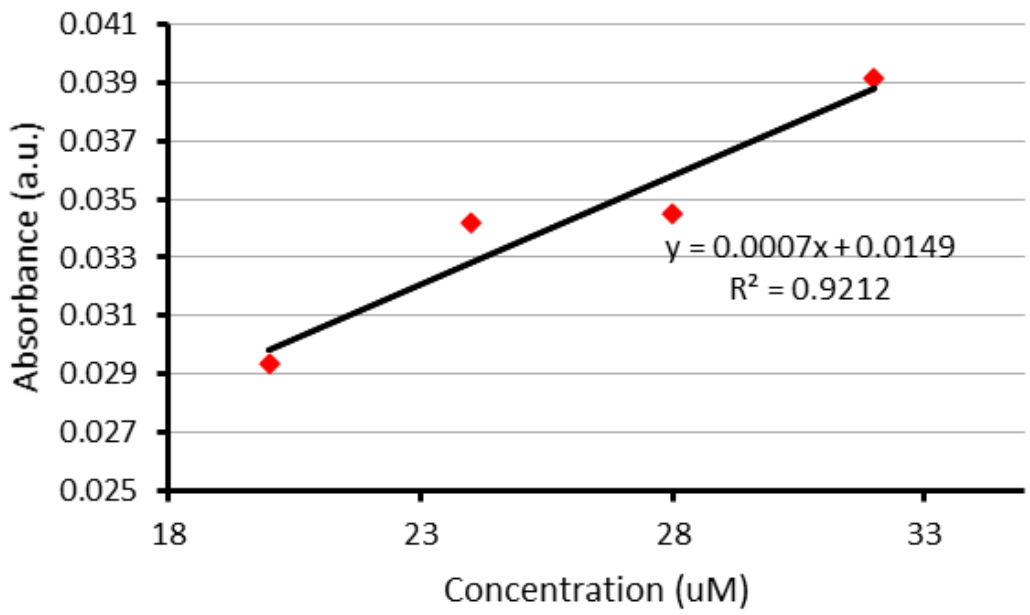


Figure S4: CP-2

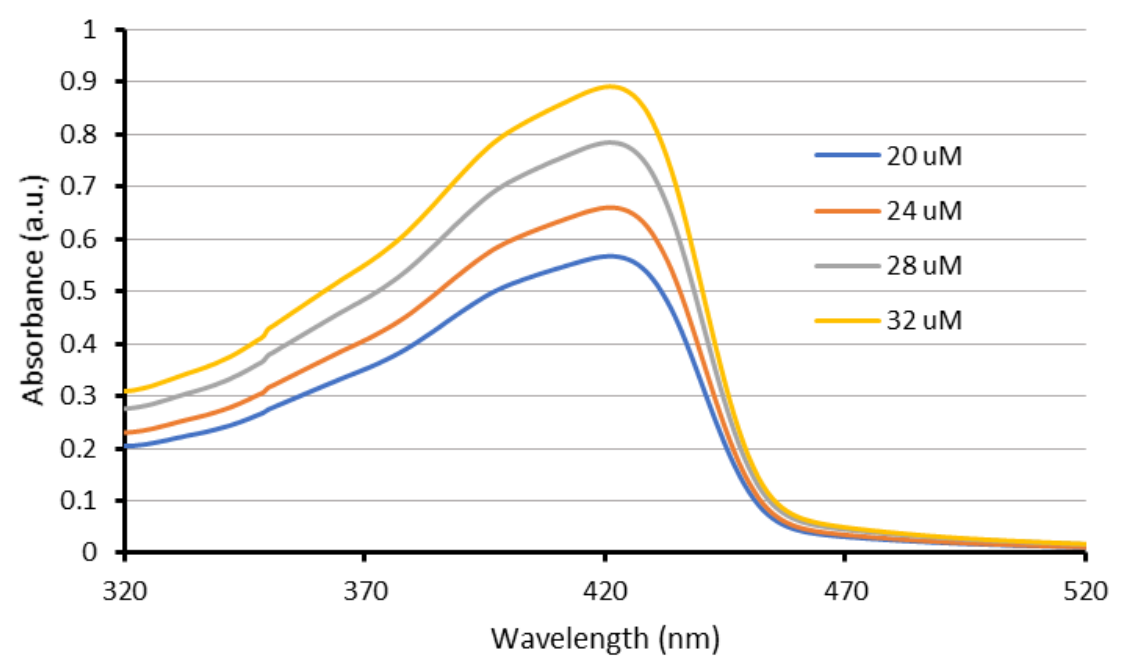

At $\lambda_{\max }$

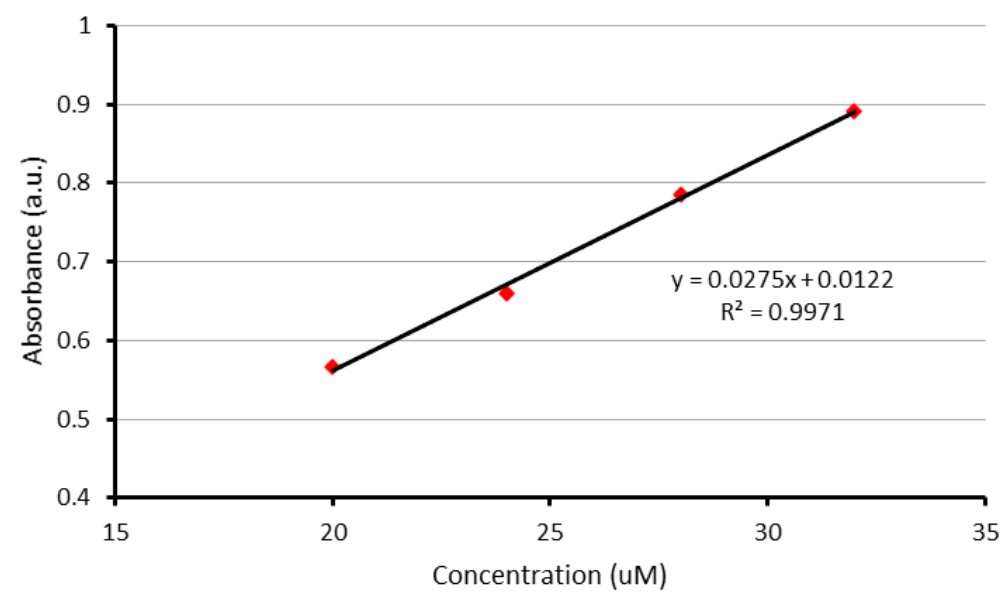

\section{At 472 nm}

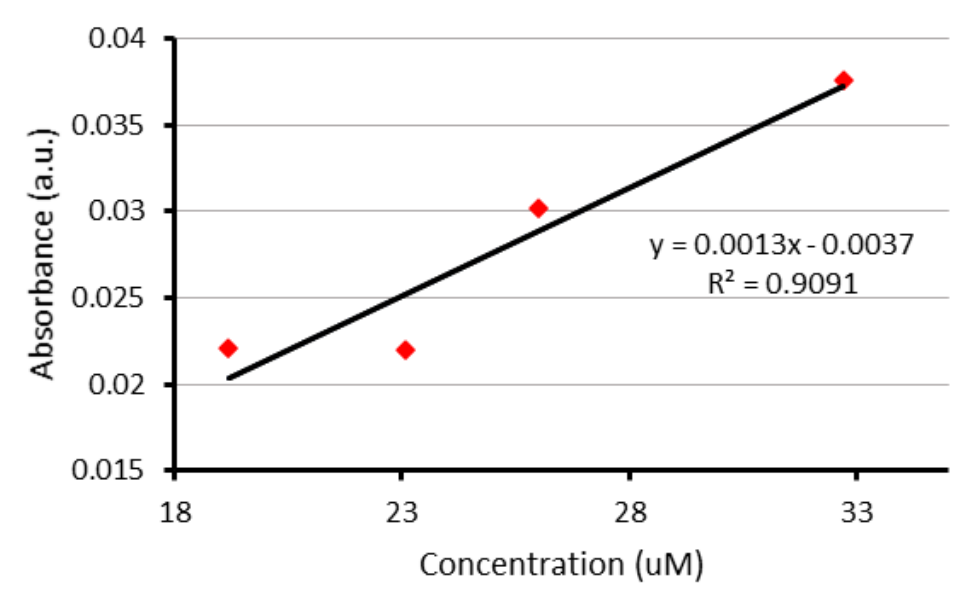


Figure S5: CP-3

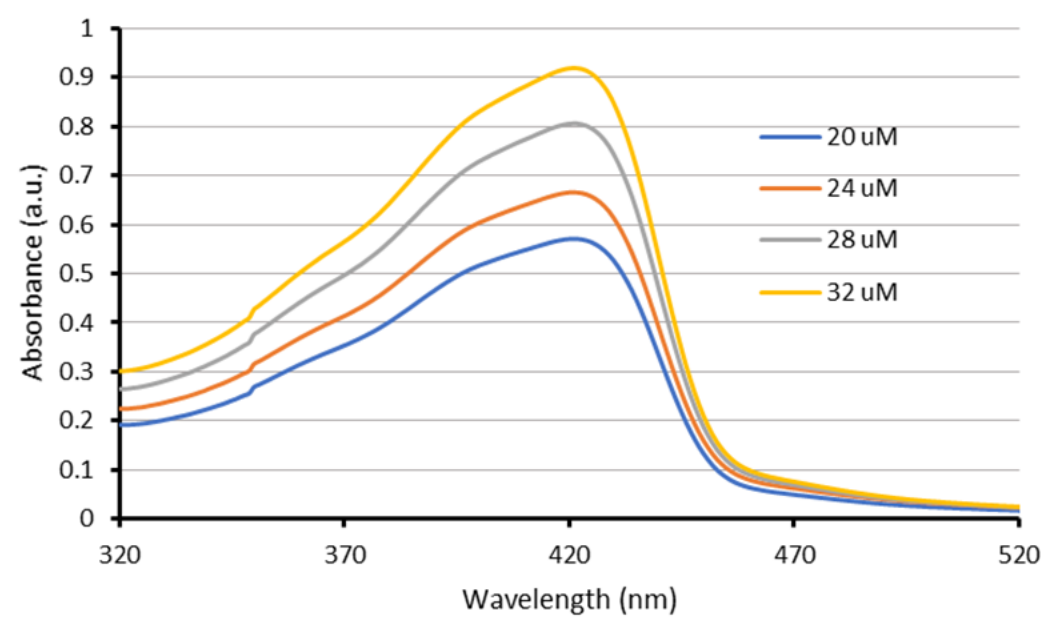

At $\lambda_{\text {max }}$

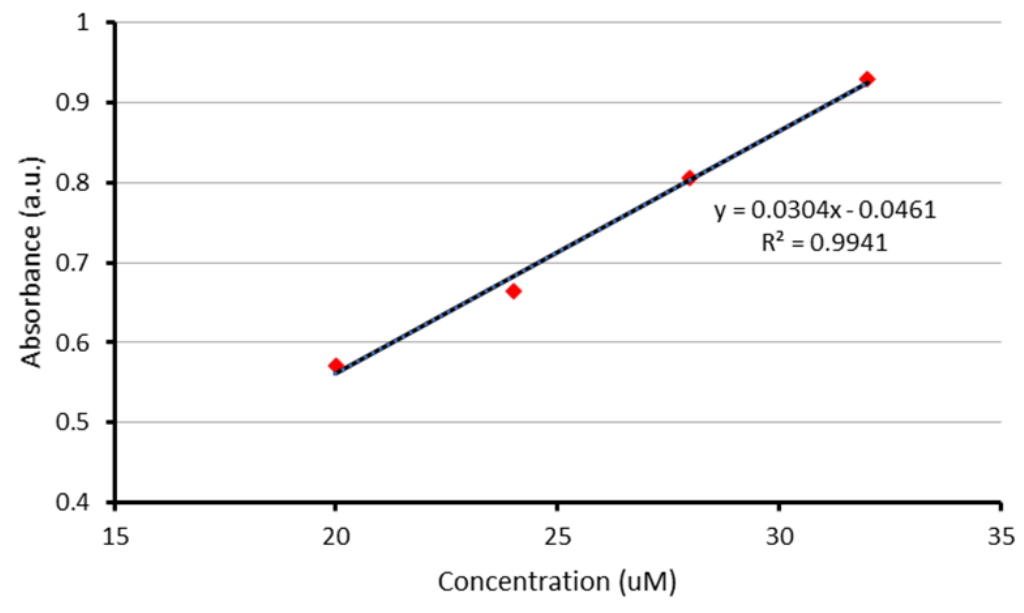

At 472 nm

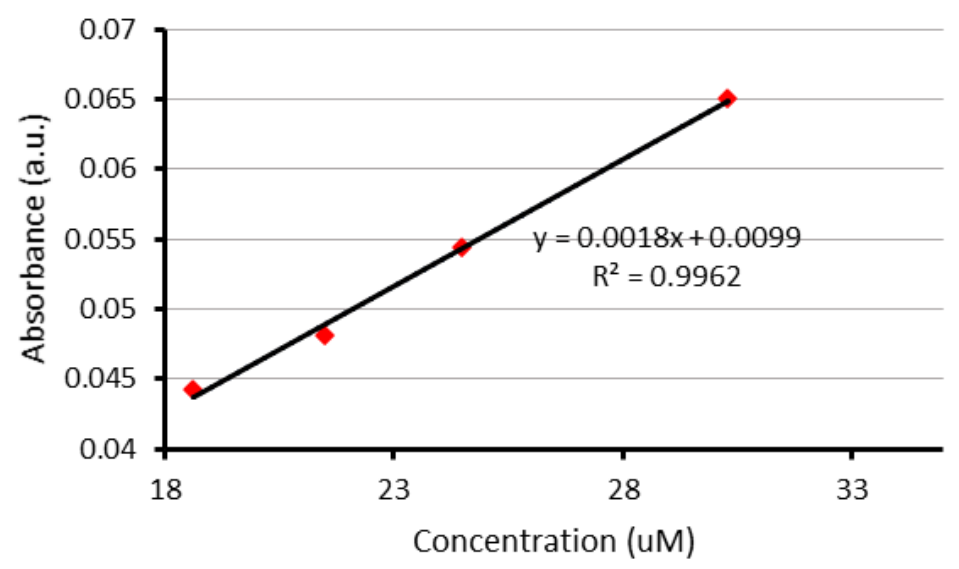


Figure S6: CP-4

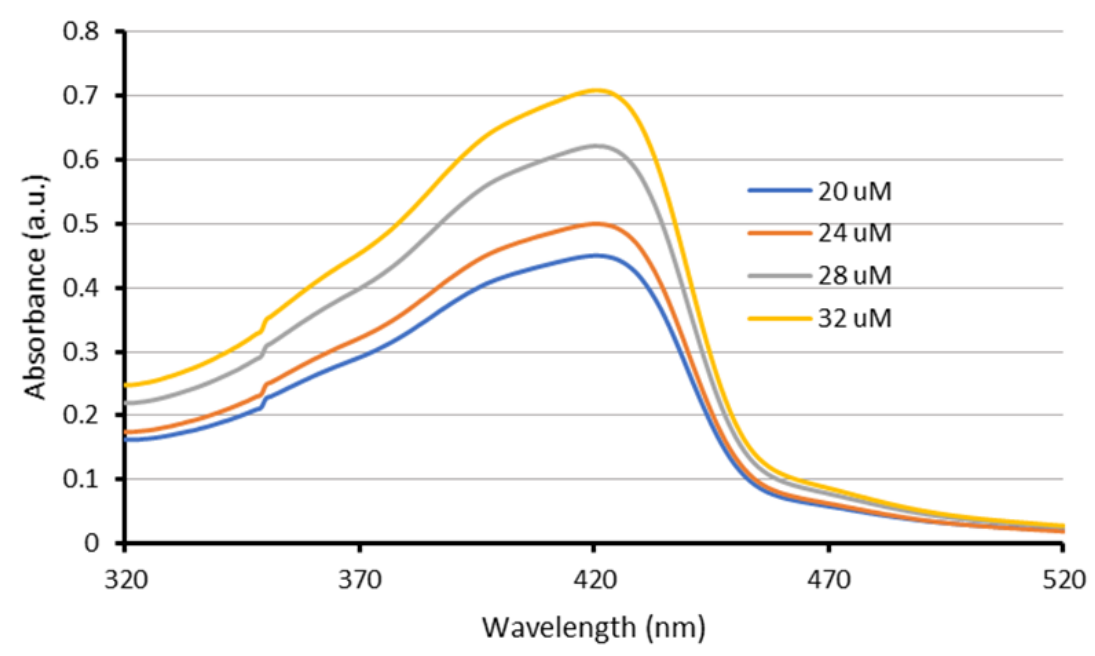

At $\lambda_{\text {max }}$

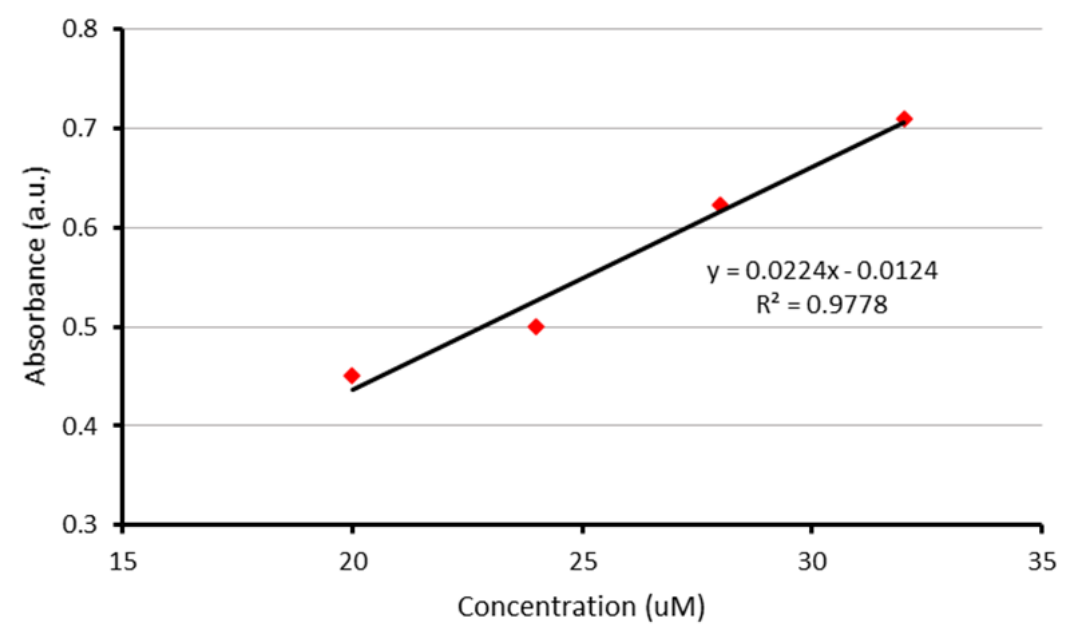

At 472 nm

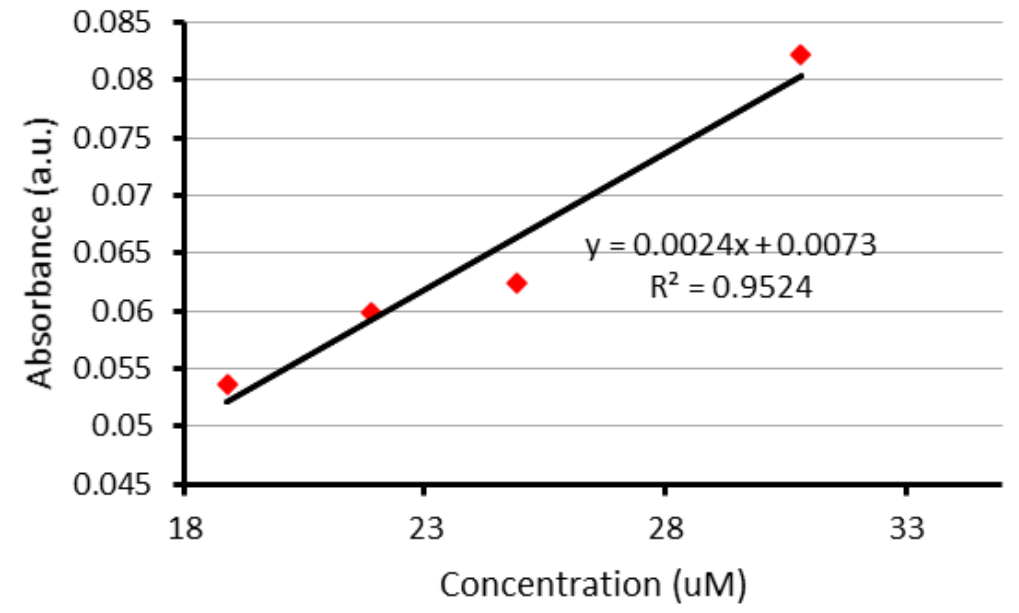




\subsection{Thin-Film Absorption Spectrum}

Figure S7: Thin film UV-Vis of copolymers

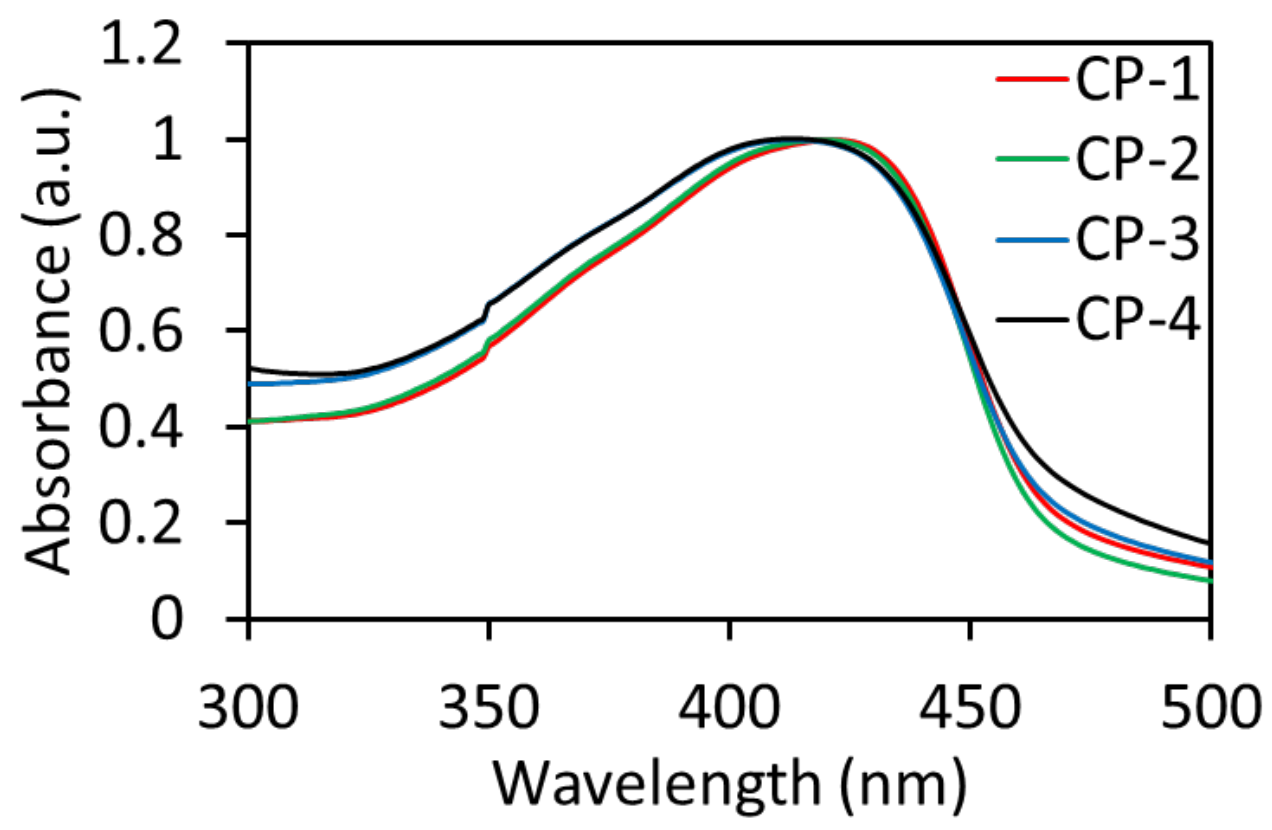

\subsection{Non-Normalized Emission Spectrum}

Figure S8

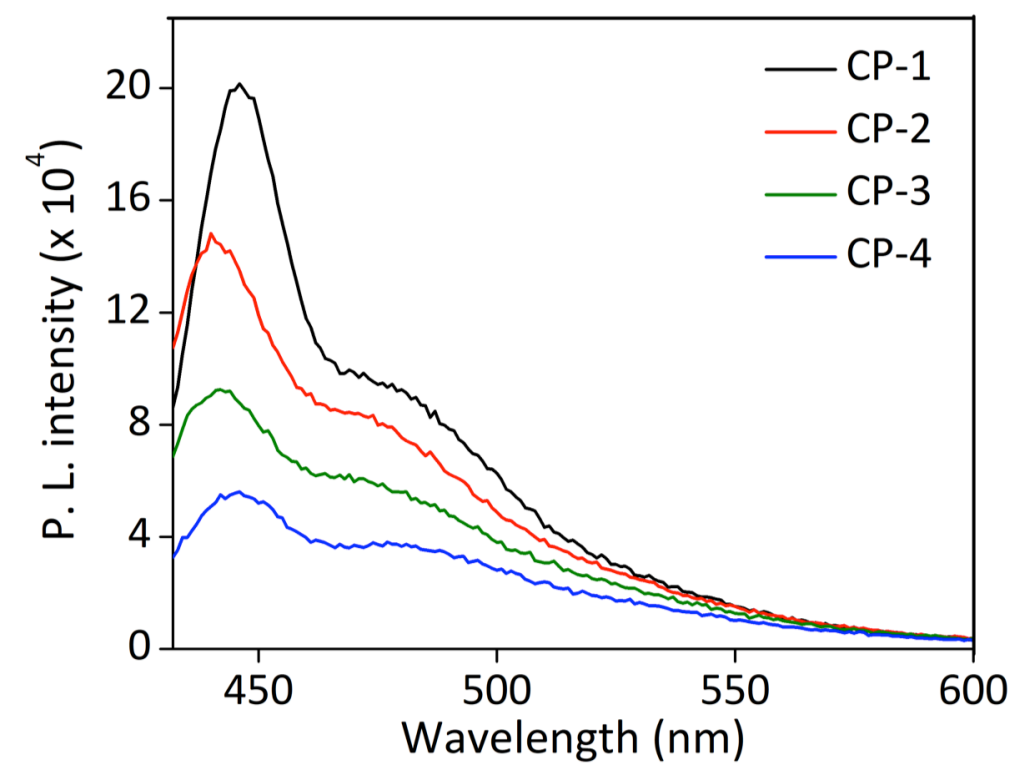

Emission spectrum of copolymers at $2.8 \mu \mathrm{M}$ in $\mathrm{CHCl}_{3}$ 


\subsection{Quantum Yield Measurements}

Figure S9: CP-1

CP-1

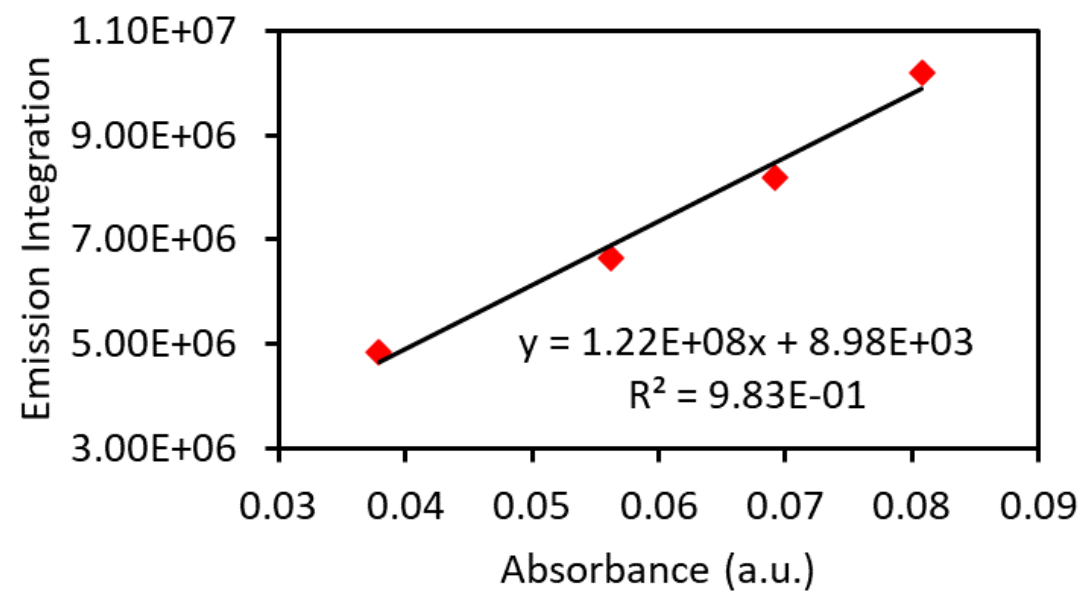

Reference

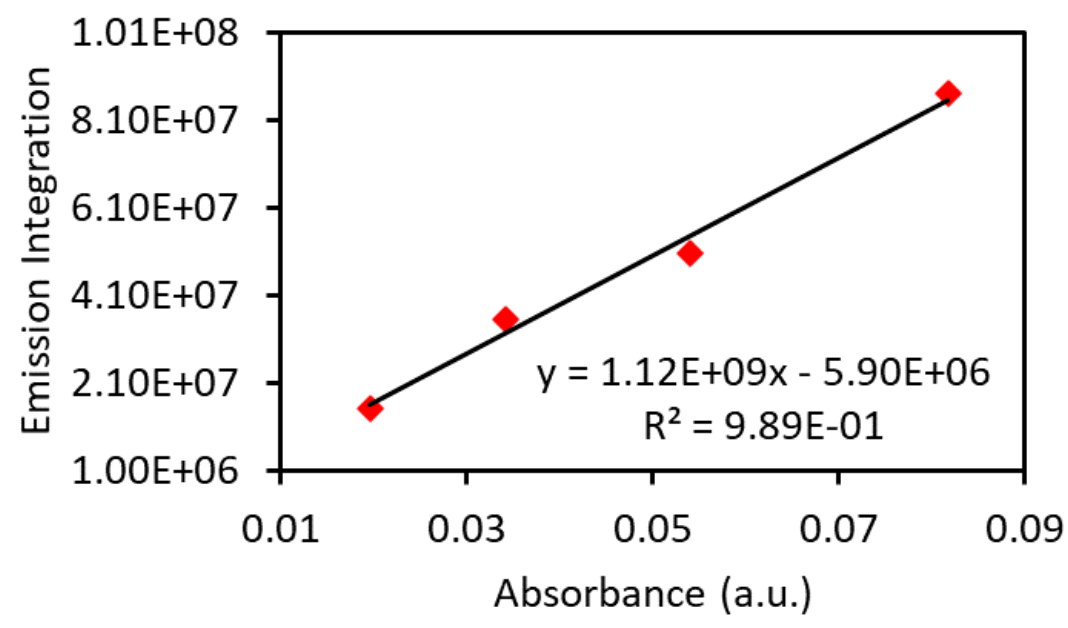


Figure S10: CP-2

CP-2

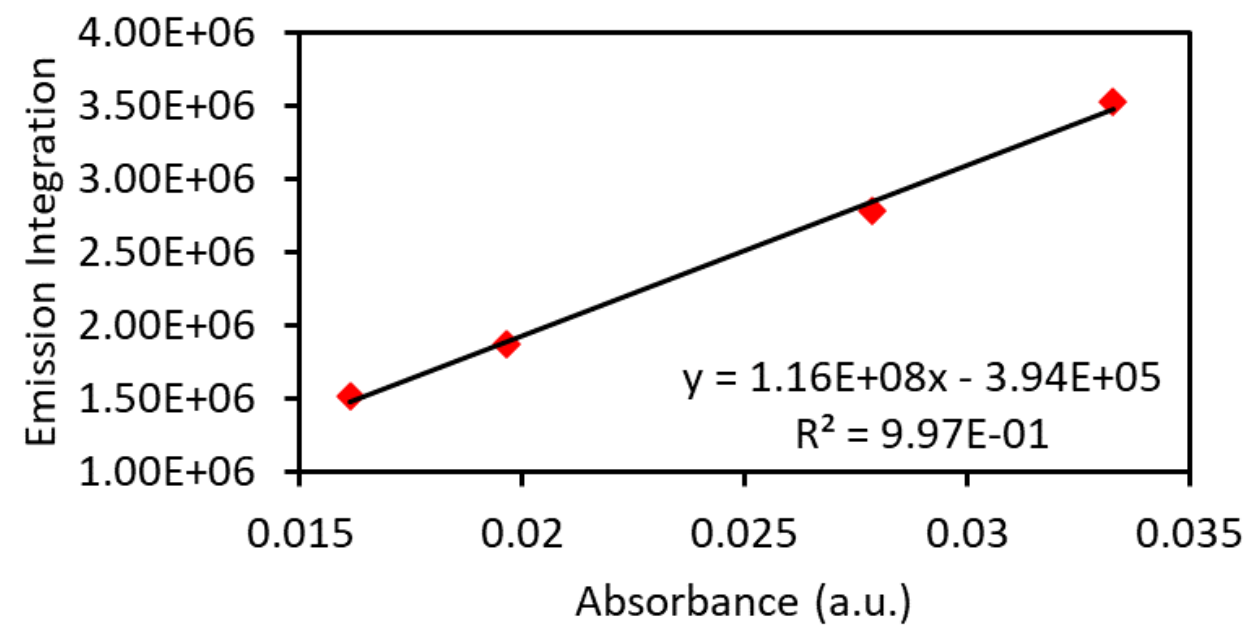

Reference

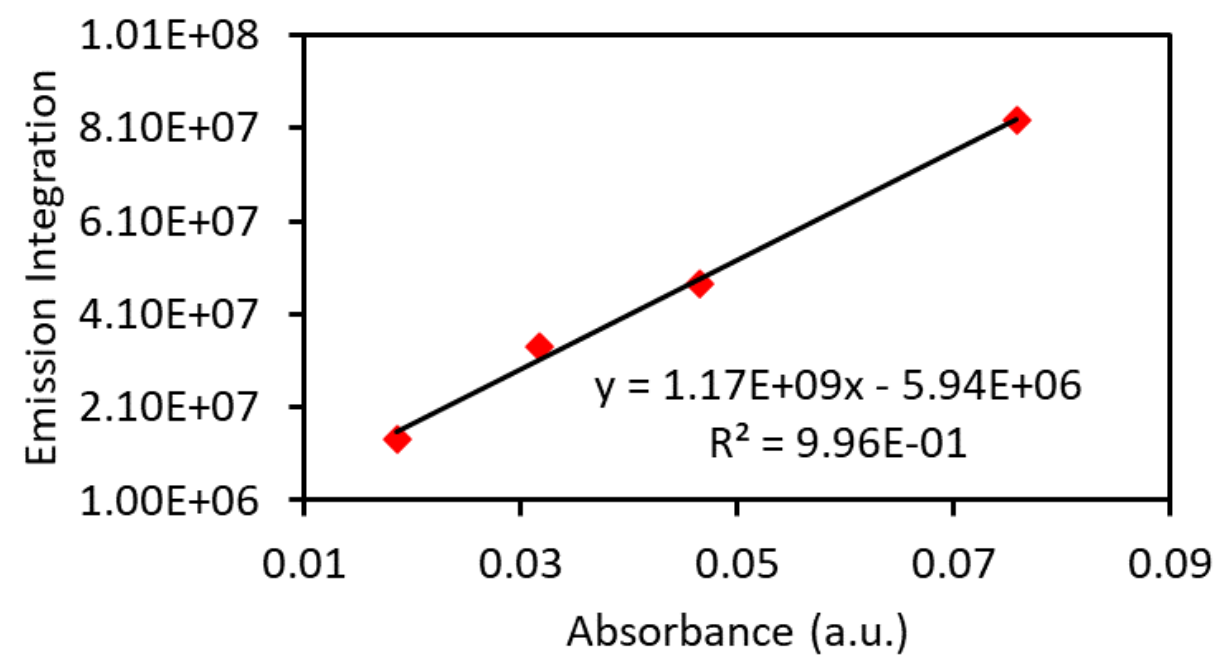


Figure S11: CP-3

CP-3

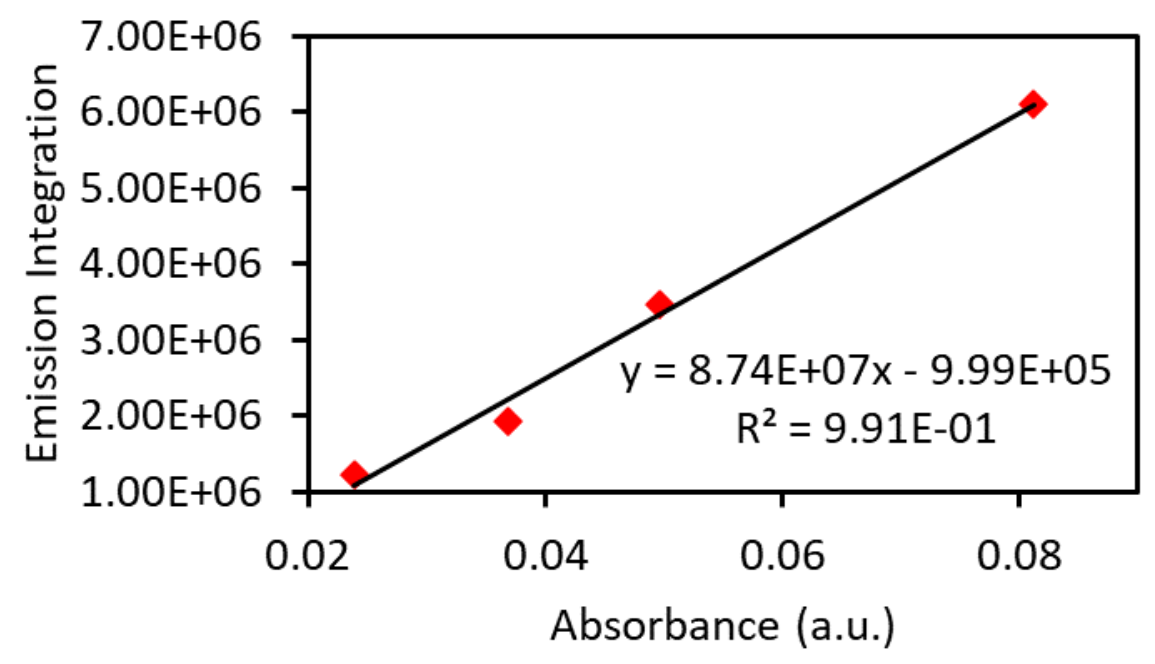

Reference

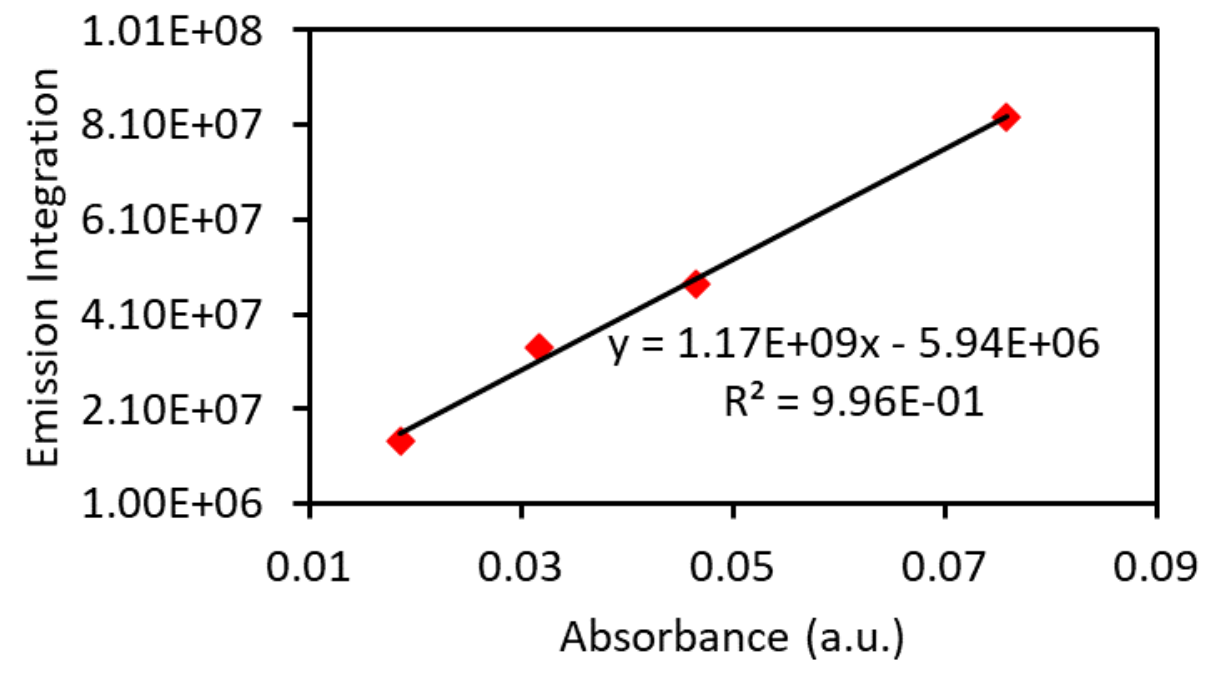


Figure S12: CP-4

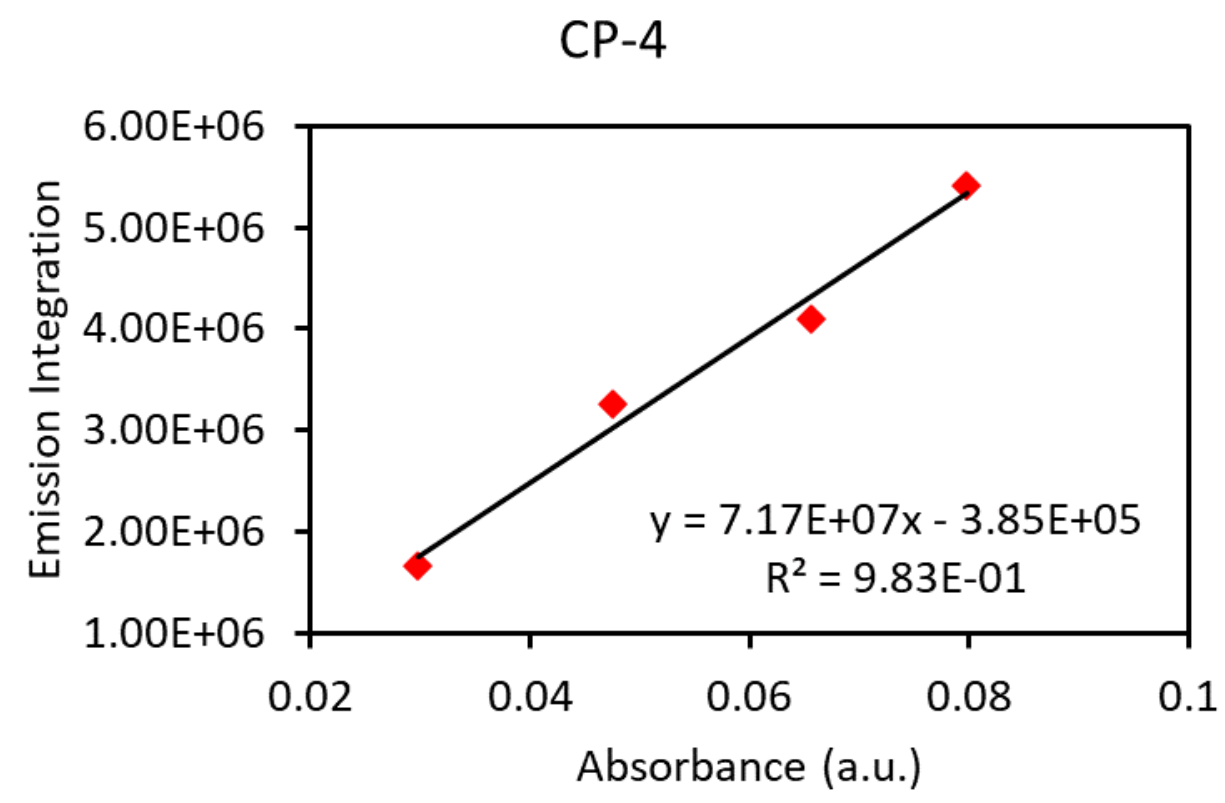

Reference

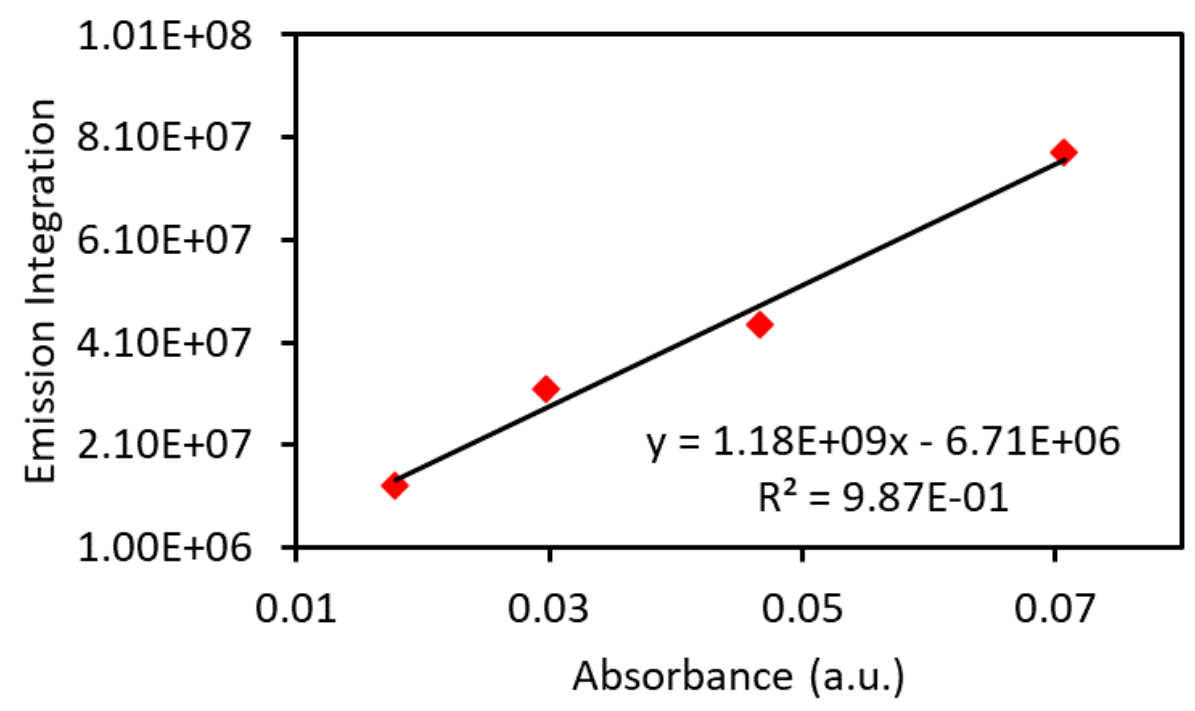


Figure S13: Adamantane Homopolymer

\section{Adamantane Homopolymer}

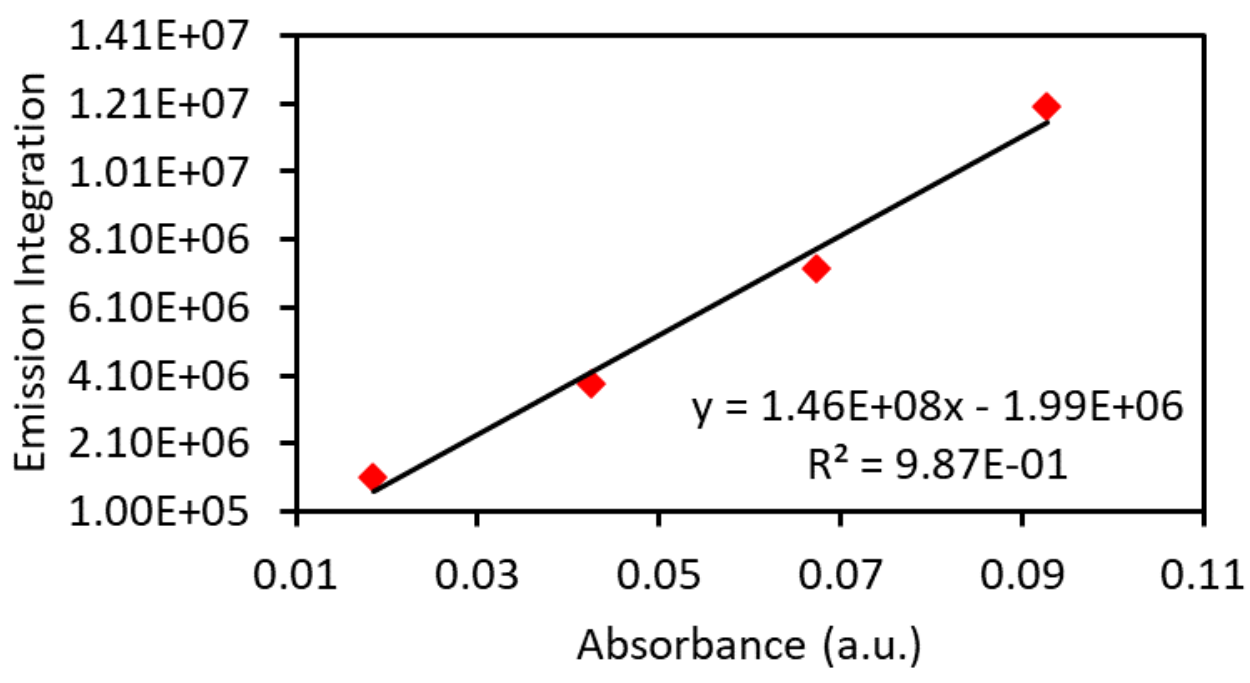

Reference

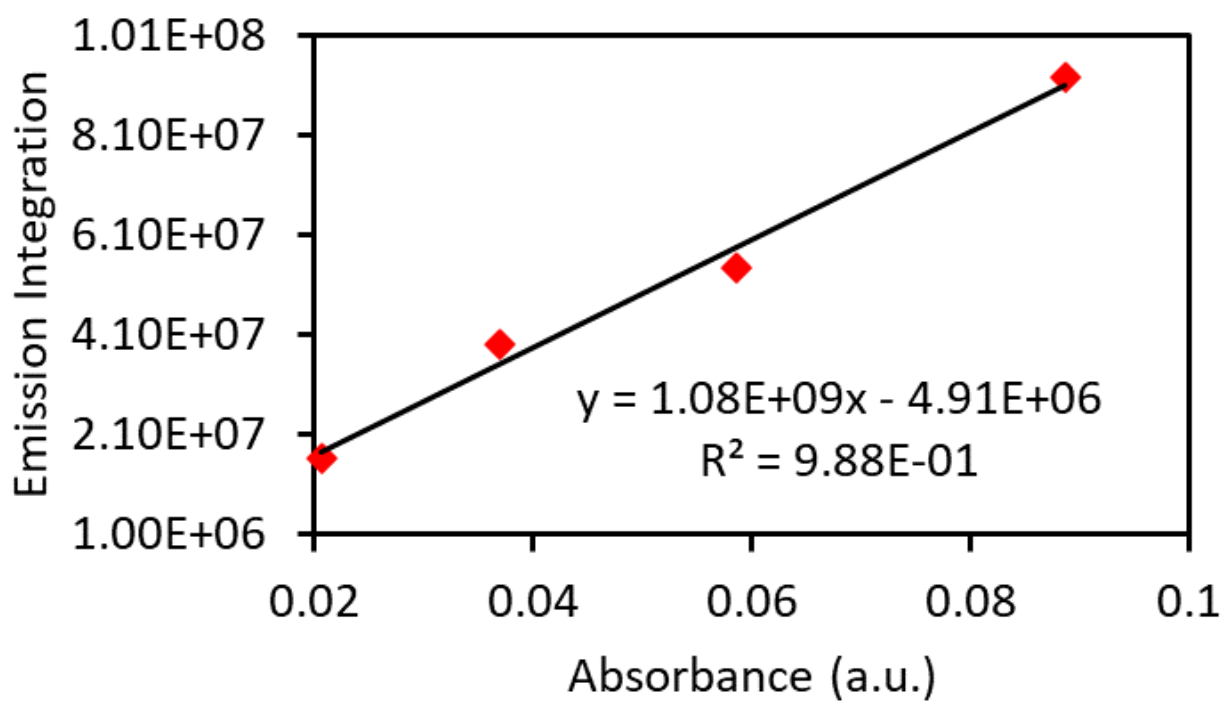


10.0 Stern-Volmer Plots of Copolymers

Figure S14: CP-1

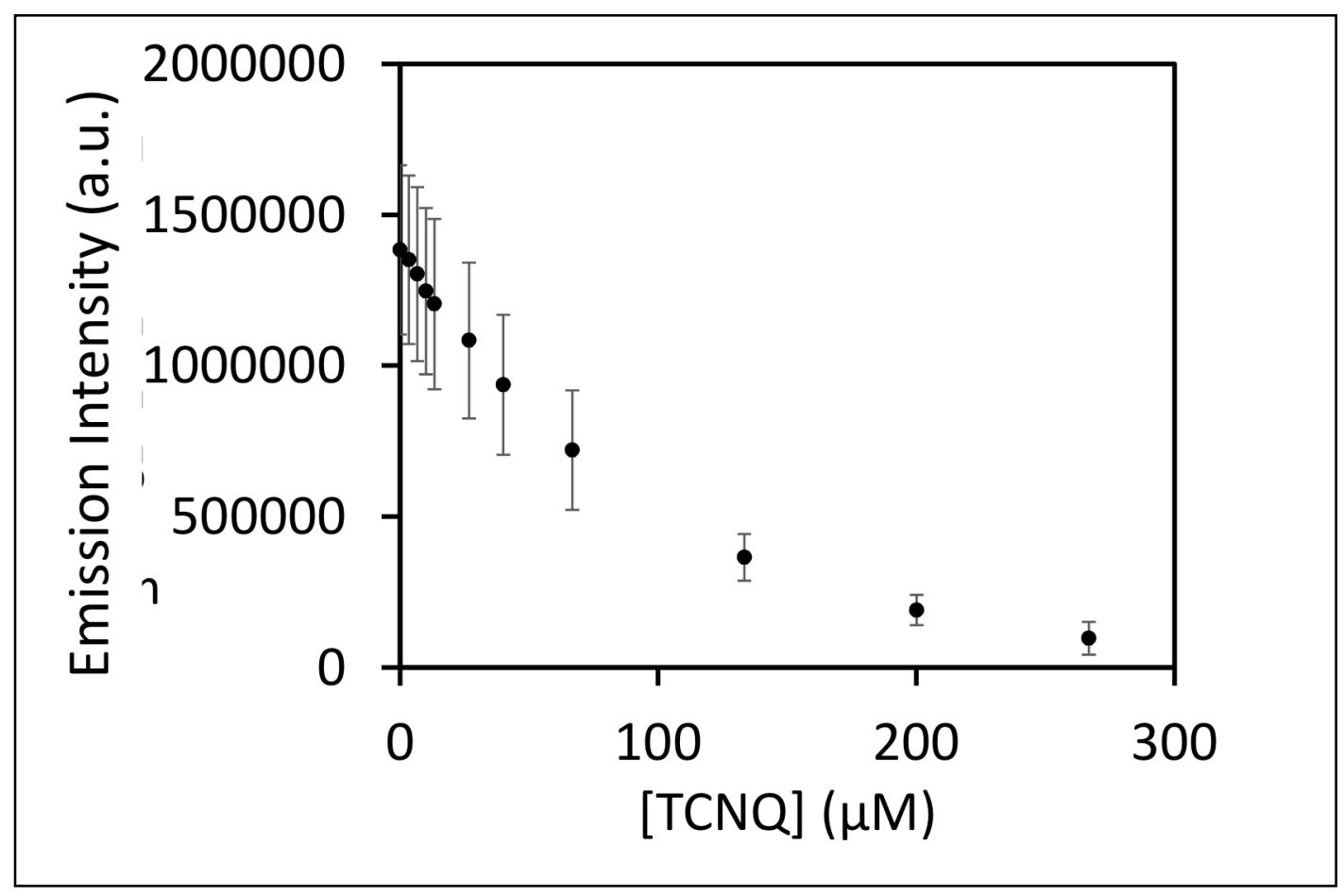

Figure S15: CP-2

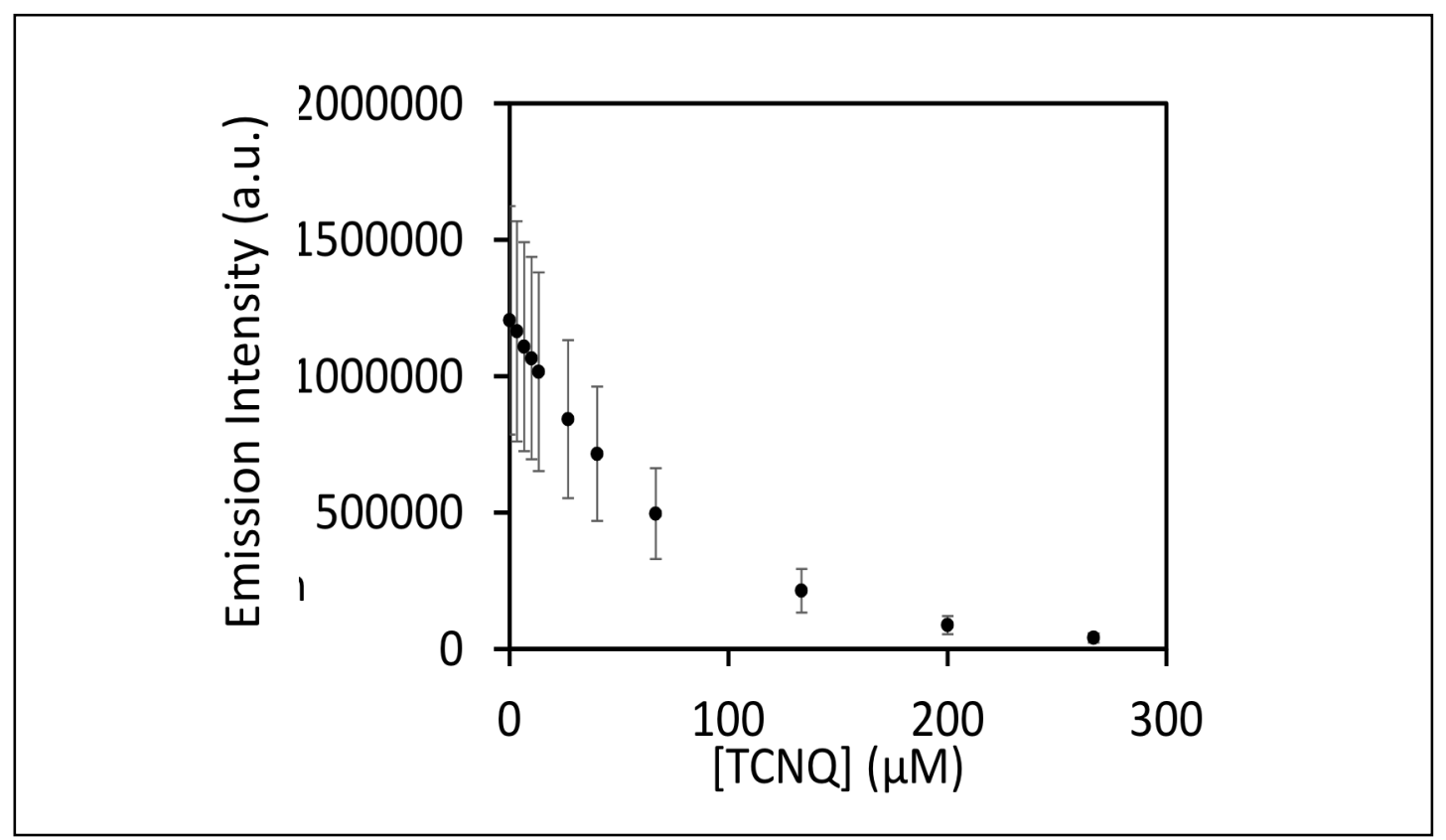


Figure S16: CP-3

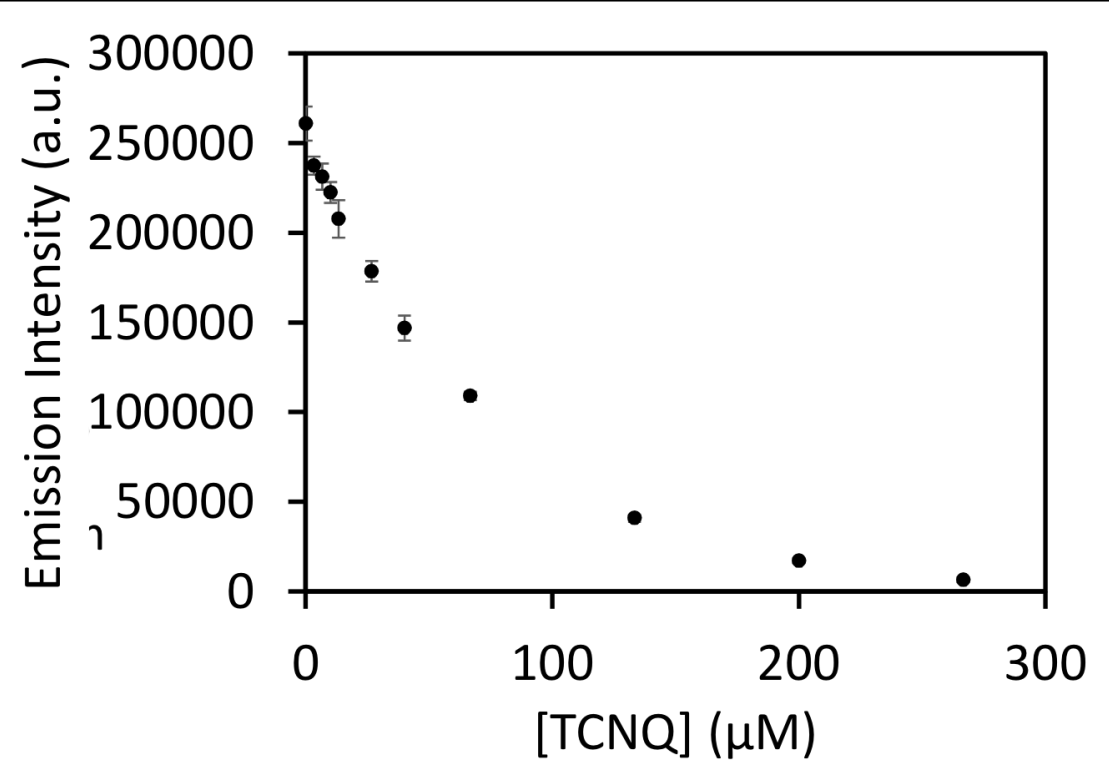

Figure S17: CP-4

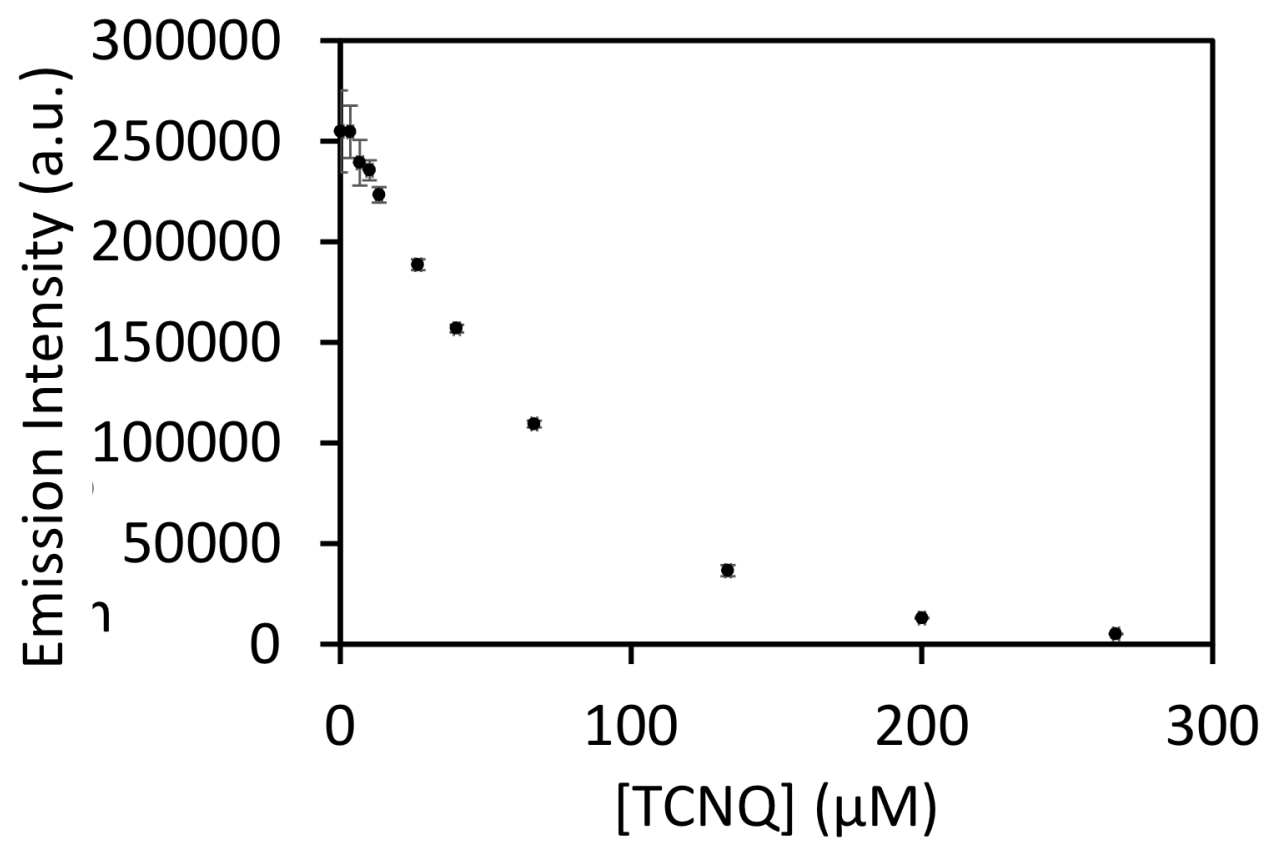




\subsection{Atomic Coordinates of ACP-PCP-ACP Trimer}

ACP-PCP-ACP trimer contains no imaginary frequencies

Table S1: Atomic Coordinates

\begin{tabular}{|r|l|r|r|r|}
\hline Atom \# & Symbol & X & Y & Z \\
\hline 1 & C & 0.702384 & 1.235813 & 0.46751 \\
\hline 2 & C & -0.7028 & -1.24293 & 0.448835 \\
\hline 3 & C & -0.68641 & 1.193228 & 0.525572 \\
\hline 4 & C & 1.410316 & 0.007971 & 0.542499 \\
\hline 5 & C & 0.685986 & -1.20122 & 0.507705 \\
\hline 6 & C & -1.41074 & -0.01634 & 0.542091 \\
\hline 7 & H & -1.24693 & 2.123738 & 0.527974 \\
\hline 8 & H & 1.246519 & -2.13166 & 0.496236 \\
\hline 9 & C & -1.40318 & -2.56748 & 0.272914 \\
\hline 10 & H & -2.18347 & -2.70486 & 1.03004 \\
\hline 11 & H & -0.68718 & -3.38753 & 0.382429 \\
\hline 12 & S & -2.36509 & -2.81597 & -1.29756 \\
\hline 13 & C & -1.14038 & -2.65799 & -2.67712 \\
\hline 14 & H & -1.73457 & -2.98448 & -3.5399 \\
\hline 15 & H & -0.34013 & -3.3938 & -2.54562 \\
\hline 16 & C & -0.56315 & -1.27437 & -2.88615 \\
\hline 17 & C & 0.564381 & 1.317934 & -2.86689 \\
\hline 18 & C & 0.823741 & -1.09185 & -2.94847 \\
\hline 19 & C & -1.38083 & -0.14 & -2.94466 \\
\hline 20 & C & -0.82256 & 1.136376 & -2.93024 \\
\hline 21 & C & 1.38199 & 0.184588 & -2.94398 \\
\hline 22 & H & 1.480171 & -1.95954 & -2.9371 \\
\hline 23 & H & -2.46072 & -0.25362 & -2.90965 \\
\hline 24 & H & -1.47896 & 2.003775 & -2.90456 \\
\hline 25 & H & 2.461937 & 0.297676 & -2.90884 \\
\hline 26 & C & 1.142006 & 2.698009 & -2.63693 \\
\hline 27 & H & 0.342041 & 3.432185 & -2.49496 \\
\hline 28 & H & 1.737023 & 3.037047 & -3.49428 \\
\hline 29 & S & 2.365853 & 2.834515 & -1.25426 \\
\hline 30 & C & 1.402818 & 2.562851 & 0.311643 \\
\hline 31 & H & 0.686698 & 3.381165 & 0.432743 \\
\hline 32 & H & 2.182555 & 2.689066 & 1.071285 \\
\hline 33 & C & 2.824038 & -0.03635 & 0.628879 \\
\hline 34 & C & 4.039358 & -0.12145 & 0.737396 \\
\hline & & 5.389362 & -0.20299 & 0.855672 \\
\hline 36.605352 & -0.27554 & 0.961871 \\
\hline C & 8.018937 & -0.35183 & 1.084679 \\
\hline
\end{tabular}




\begin{tabular}{|r|l|r|r|r|}
\hline 38 & $\mathrm{C}$ & 10.79731 & -0.42968 & 1.318526 \\
\hline 39 & $\mathrm{C}$ & 8.658558 & -1.59965 & 1.289904 \\
\hline 40 & $\mathrm{C}$ & 8.785342 & 0.829355 & 1.000823 \\
\hline 41 & $\mathrm{C}$ & 10.17426 & 0.80992 & 1.115038 \\
\hline 42 & $\mathrm{C}$ & 10.05093 & -1.60399 & 1.402868 \\
\hline 43 & $\mathrm{H}$ & 8.267831 & 1.771408 & 0.842497 \\
\hline 44 & $\mathrm{H}$ & 10.56208 & -2.55073 & 1.561263 \\
\hline 45 & $\mathrm{H}$ & 11.88018 & -0.47684 & 1.412197 \\
\hline 46 & $\mathrm{C}$ & 7.860289 & -2.87519 & 1.383113 \\
\hline 47 & $\mathrm{H}$ & 7.280623 & -3.05213 & 0.46903 \\
\hline 48 & $\mathrm{H}$ & 7.139103 & -2.83651 & 2.208479 \\
\hline 49 & $\mathrm{H}$ & 8.515227 & -3.737 & 1.543155 \\
\hline 50 & $\mathrm{C}$ & 10.98626 & 2.080935 & 1.020413 \\
\hline 51 & $\mathrm{H}$ & 11.68023 & 2.049348 & 0.171177 \\
\hline 52 & $\mathrm{H}$ & 11.58958 & 2.238058 & 1.922972 \\
\hline 53 & $\mathrm{H}$ & 10.34339 & 2.957112 & 0.892021 \\
\hline 54 & $\mathrm{C}$ & -2.82448 & 0.026718 & 0.628749 \\
\hline 55 & $\mathrm{C}$ & -4.03983 & 0.110224 & 0.738186 \\
\hline 56 & $\mathrm{C}$ & -5.38986 & 0.19006 & 0.857309 \\
\hline 57 & $\mathrm{C}$ & -6.60587 & 0.261078 & 0.964285 \\
\hline 58 & $\mathrm{C}$ & -8.01946 & 0.335613 & 1.088147 \\
\hline 59 & $\mathrm{C}$ & -10.798 & 0.410474 & 1.320851 \\
\hline 60 & $\mathrm{C}$ & -8.65982 & 1.581664 & 1.301739 \\
\hline 61 & $\mathrm{C}$ & -8.78521 & -0.84534 & 0.995778 \\
\hline 62 & $\mathrm{C}$ & -10.1741 & -0.82751 & 1.110214 \\
\hline 63 & $\mathrm{C}$ & -10.0523 & 1.584556 & 1.4137 \\
\hline 64 & $\mathrm{H}$ & -8.26721 & -1.7859 & 0.830292 \\
\hline 65 & $\mathrm{H}$ & -10.5641 & 2.530094 & 1.577064 \\
\hline 66 & $\mathrm{H}$ & -11.881 & 0.456601 & 1.41297 \\
\hline 67 & $\mathrm{C}$ & -10.9847 & -2.09935 & 1.014638 \\
\hline 68 & $\mathrm{H}$ & -11.7411 & -2.03292 & 0.223045 \\
\hline 69 & $\mathrm{H}$ & -11.5171 & -2.30508 & 1.95176 \\
\hline 70 & $\mathrm{H}$ & -10.3489 & -2.96301 & 0.797291 \\
\hline 71 & $\mathrm{C}$ & -7.86232 & 2.857072 & 1.403105 \\
\hline & $\mathrm{H}$ & -7.28466 & 3.041483 & 0.489213 \\
\hline & -7.13943 & 2.812596 & 2.226662 \\
\hline 73 & -8.51759 & 3.717125 & 1.571027 \\
\hline
\end{tabular}




\section{$12 .{ }^{1} \mathrm{H}$ NMR Spectra}
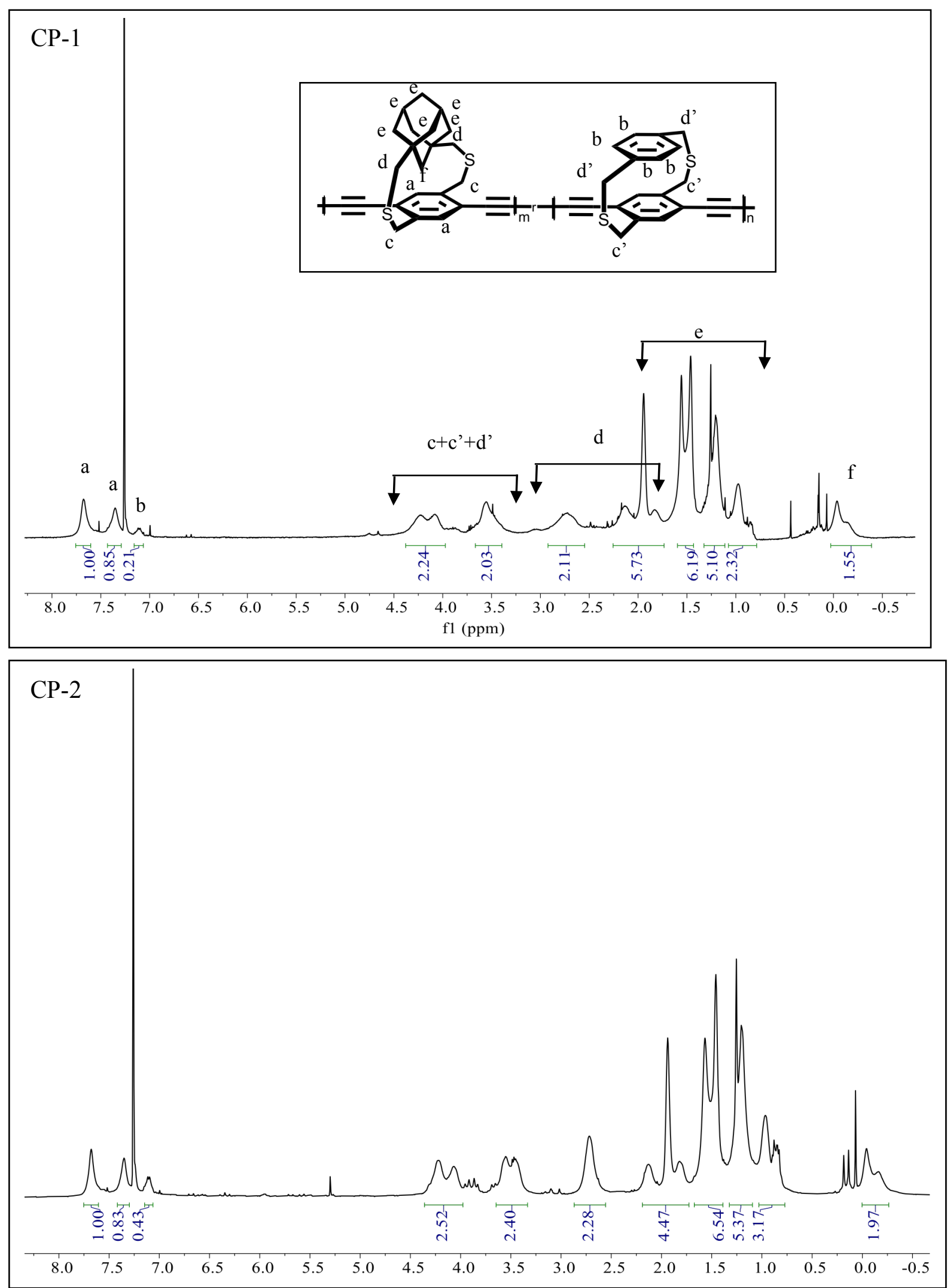

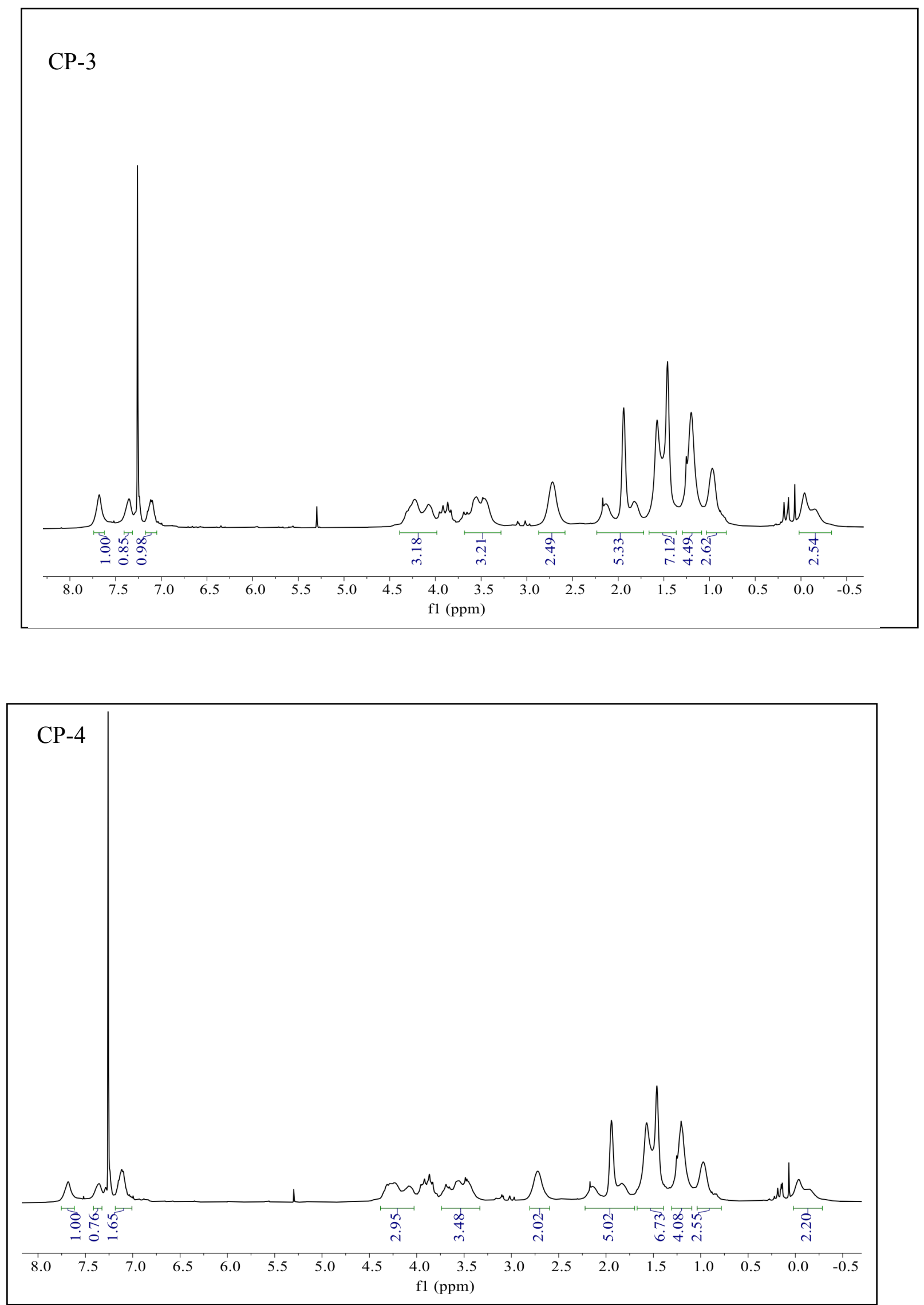